\title{
Spatial Utilization and Microhabitat Selection of the Snow Leopard (Panthera uncia) under Different Livestock Grazing Intensities
}

\author{
Yang Hong, ${ }^{\mathrm{a}}$ Thomas Connor, ${ }^{\mathrm{b}}$ Huan Luo,${ }^{\mathrm{a}}$ Xiaoxing Bian, ${ }^{\mathrm{c}}$ Zhaogang Duan, ${ }^{\mathrm{d}}$ Zhuo Tang, ${ }^{\mathrm{d}}$ \\ AND JINDONG ZHANG ${ }^{\mathrm{a}}$ \\ ${ }^{a}$ Key Laboratory of Southwest China Wildlife Resources Conservation, Ministry of Education, China West Normal University, \\ Nanchong, Sichuan Province, China \\ ${ }^{\mathrm{b}}$ Department of Environmental Science, Policy, and Management, University of California, Berkeley, Berkeley, California \\ ${ }^{\mathrm{c}}$ Department of Wildlife Ecology and Conservation, University of Florida, Gainesville, Florida \\ ${ }^{\mathrm{d}}$ Administration Bureau of Wolong National Nature Reserve, Aba, China
}

(Manuscript received 1 February 2021, in final form 22 July 2021)

\begin{abstract}
There is increasing conflict between snow leopards and humans in many protected areas, the main driver of which is the overlap in spatial utilization between snow leopards and livestock. Understanding the spatial utilization and microhabitat selection of snow leopards in areas featuring different levels of livestock grazing is important to better understand and resolve this conflict, but such studies are rare. Here, we conducted line transect and plot surveys in low- and high-grazing-disturbance areas (LGDAs and HGDAs) in Wolong National Reserve, southwestern China. We compared snow leopard spatial utilization and microhabitat characteristics between LGDAs and HGDAs. Results showed that snow leopards had aggregated distribution in both LGDAs and HGDAs, but the distribution of snow leopards in HGDAs was more centralized than in LGDAs. Herb cover and height in LGDAs were greater than in HGDAs. We fit a resource selection function (RSF) that showed that snow leopards preferentially selected higher elevation, smaller basal diameter of shrubs, and lower height of herbs in LGDAs. In contrast, there were no significant microhabitat factors in our snow leopard RSF in HGDAs. Our results indicate that high-intensity grazing tends to reduce the habitat types available to and preferential selectivity of habitat by snow leopards. We recommend that livestock grazing should be controlled to restore the diversity of the alpine ecosystems in Wolong Nature Reserve. Our findings also highlight the need for evaluating the impact of livestock grazing on rare animals in alpine environments (e.g., snow leopard) in other areas facing similar issues.
\end{abstract}

KEYWORDS: Asia; Animal studies; Ecosystem effects; Local effects

\section{Introduction}

With the continued development of human society, there are increasing conflicts between humans and wildlife (Rovero et al. 2020; Berger et al. 2013). Some of the most severe examples of this conflict occur between humans and large carnivores (Dickman 2009; Koziarski et al. 2016). A key driver for this conflict is domestic livestock taking up the habitat and food resources of wild ungulates, which in turn affects the survival of large carnivores (Suryawanshi et al. 2017). Research by Sharma et al. (2015) indicated that snow leopards and livestock can coexist under a certain threshold of livestock density. But, when the density is higher than this threshold, the habitat use of snow leopards will decline in the area (Sharma et al. 2015). Additionally, as wild ungulate populations decline, large carnivores begin to prey on livestock (Landa et al. 1999; Morell 2017; Suryawanshi et al. 2013). As livestock are preyed upon by large carnivores, potentially large economic losses are faced by local farmers, who may carry out retaliatory killings of large predators (Johansson et al. 2015; Bagchi and Mishra 2006). The interaction among snow leopard, pastoralists, and their

Corresponding author: Jindong Zhang, zhangjd224@163.com

Earth Interactions is published jointly by the American Meteorological Society, the American Geophysical Union, and the Association of American Geographers. livestock in Asia is a prime example of this system of human and large carnivore conflict.

Although the snow leopard was downlisted from endangered (EN) to vulnerable (VU) on the Red List of Threatened Species by the International Union for Conservation of Nature (IUCN) in 2017 (McCarthy et al. 2017), snow leopard populations have been declining and their suitable habitat has been shrinking (Liu and Han 2015). There are many factors contributing to this trend, including human disturbance and climate change (Jessica et al. 2012; Farrington and Li 2016; Alexander et al. 2016a). Livestock grazing is one of the major human disturbances throughout the snow leopard's range. Understanding the impact of livestock on snow leopard habitat is an important consideration for their conservation. There has been extensive research on the effect of livestock on many wildlife species such as the giant panda (Ailuropoda melanoleuca) (Wang et al. 2019; Hull et al. 2014; Zhang et al. 2017; Wei et al. 2018), the red panda (Ailurus fulgens) (Wang et al. 2018a), and the tiger (Panthera tigers) (Bargali et al. 2018; B. B. Li et al. 2020). Through the above studies, we hypothesize that the increasing livestock pressure will also negatively influence the ecology-spatial utilization and microhabitat selection of snow leopard. To verify this hypothesis, we investigated the effect of livestock grazing on two aspects of snow leopard ecologyspatial utilization and microhabitat selection in this study.

Spatial utilization of wildlife is a function of the distribution of their population, their range of activity within that distribution, response to habitat changes, and other factors 
(Zhang et al. 2019; Bai 2017; Lukarevskiy et al. 2019). Previous research has found that the spatial ranges of livestock and snow leopards often overlap significantly, leading to high risks of snow leopard-livestock conflicts (Shi et al. 2019). Unfortunately, most current studies on the spatial distribution of snow leopards are only predictions of their distribution in a certain region based on data from another (Ghoshal et al. 2019; Thinley et al. 2014; Jackson 2002). Few studies have considered the effects of livestock grazing on distributions of snow leopards in China. Research on the habitat selection of snow leopards has mainly focused on the comparison of single habitat features between the regions where snow leopards appear and do not appear, and multiple habitat variables have not been comprehensively considered (Xu et al. 2006; Qiao et al. 2017; Sandeep et al. 2006). Additionally, previous studies on habitat selection of snow leopards have mainly focused on topographic variables (elevation, slope, etc.), ignoring detailed vegetation variables (shrub height, number of shrubs, herb coverage, etc.) that can be very important in determining wildlife habitat selection (Tang et al. 2017; Bai et al. 2020). Investigating a wider range of habitat factors will result in a better understanding of the impact of livestock on the space use and microhabitat selection of snow leopards, which will inform appropriate adjustments to the management of livestock and alleviate the conflict between human and leopards. To achieve this, we conducted surveys to record snow leopard sign in low-grazing-disturbance areas (LGDAs) and high-grazing-disturbance areas (HGDAs). We also surveyed sample plots to measure diverse habitat information (including topographic and detailed vegetation variables) to understand the difference in snow leopard microhabitat use under different grazing conditions.

\section{Study area}

We conducted our study in Wolong Nature Reserve $\left(102^{\circ} 52^{\prime}-\right.$ $103^{\circ} 24^{\prime} \mathrm{E}, 30^{\circ} 45^{\prime}-31^{\circ} 25^{\prime} \mathrm{N}$ ), which is located in Sichuan Province in southwestern China (Fig. 1). The reserve covers an area of approximately $2000 \mathrm{~km}^{2}$, with diverse habitat types including forests, meadows, and alpine screes, occurring across elevations ranging from 1190 to $6250 \mathrm{~m}$. The forest types include evergreen broadleaf forest, deciduous and evergreen broadleaved forest, deciduous broadleaved forest, mixed coniferous broadleaved forest, coniferous forest, and alpine shrubland. Common plan species in the reserve include Abies fabri (Mast.) Craib., Betula albosinensis Burk., Rhododendron L., Fargesia robusta Yi, Bashania fangiana (A. Camus) Keng f., and others. The annual average temperature is $8.7^{\circ} \mathrm{C}$, and the annual average precipitation is $890 \mathrm{~mm}$. The reserve is rich in animal and plant resources, with about 450 vertebrate species and 1898 higher plant species (Cheng et al. 2015). Rare and threatened species that occur in the park include the snow leopard, giant panda, sambar deer (Rusa Unicolor), golden snub-nosed monkey (Rhinopithecus Roxellana) (Hou et al. 2018; Shi et al. 2017), gong tong (Davidia involucrata Baillon), and shui shan (Metasequoia glyptostroboides $\mathrm{Hu}$ and W. C. Cheng) (Zhang et al. 2005). The reserve is part of the Qionglai Mountain range, which is the southeastern edge of the global distribution of snow leopards (Lu et al. 2019). The snow leopard was first detected in Wolong by infrared camera traps in 2009 (Qiao et al. 2017), and thus research into snow leopards there has only begun relatively recently. Currently there is no accurate estimate of the snow leopard population in Wolong Nature Reserve. Many reserves have only one flagship species, which provides protection to other species through its umbrella function, but studies have shown that the umbrella function of flagship species may not provide sufficient protection for other species (S. Li et al. 2020; Wang et al. 2021). Wolong Nature Reserve mainly focuses on the protection of giant pandas with little focus on the protection of snow leopards, leading to a relative lack of research on snow leopards and their alpine ecosystem. For example, research on livestock grazing effects has been concentrated in low-elevation areas within giant panda habitat (Wang et al. 2019), while there has been no research on the effects of livestock grazing on the ecology and conservation of snow leopards in Wolong Nature Reserve.

Snow leopards are mainly distributed in the Yinchanggou, Weijiagou, and Tizigou regions in the southwest of Wolong Nature Reserve (Tang et al. 2017), where livestock are widely distributed as well. We focused on Yinchanggou and Weijiagou as our research areas, where the density of livestock signs is 0.17 and $5.84 \mathrm{~km}^{2}$, respectively. Wolong Nature Reserve had 5000 permanent residents in 2009 (Liu et al. 2009), and their main source of income has traditionally been livestock grazing and farming (Hull et al. 2014; Zhang et al. 2017). Although tourism has become a bigger part of the local population's income, the number of livestock in the reserve has been increasing in recent years (Wang et al. 2018b; Zhang et al. 2018). Many livestock are grazed in the alpine meadow ecosystem in Wolong Nature Reserve, where they compete with blue sheep (Pseudois nayaur), goral (Naemorhedus), marmot (Marmota), and other herbivores (Lu et al. 2019).

\section{Methods}

\section{a. Data collection}

We used results from a questionnaire survey administered to 202 local farmers on the distribution of livestock in Wolong Nature Reserve (Wang et al. 2018b) to define HGDAs versus LGDAs. Specifically, we defined Weijiagou as an HGDA because of a relatively high livestock sign density of 5.84 animals $\mathrm{km}^{-2}$ and defined Yinchangou as an LGDA because of its relatively low livestock sign density of 0.17 animals $\mathrm{km}^{-2}$ (Fig. 1). The elevation span and vegetation communities of two mesoscale study areas (Yinchanggou and Weijiagou) are similar. Additionally, the two areas are not far apart and have no significant barriers in between, so the wildlife in the two areas are not isolated from each other. Therefore, the comparison of livestock effects between the two areas is robust. To sample for snow leopard presence and habitat characteristics, we used line transect and sample plot methods. We set up line transects in a systematically random manner, with each line transect at least $3 \mathrm{~km}$ in length and separated by at least $500 \mathrm{~m}$. The research team walked the line transects to find signs of snow leopard (e.g., feces and footprint) with the help of experienced guides. 


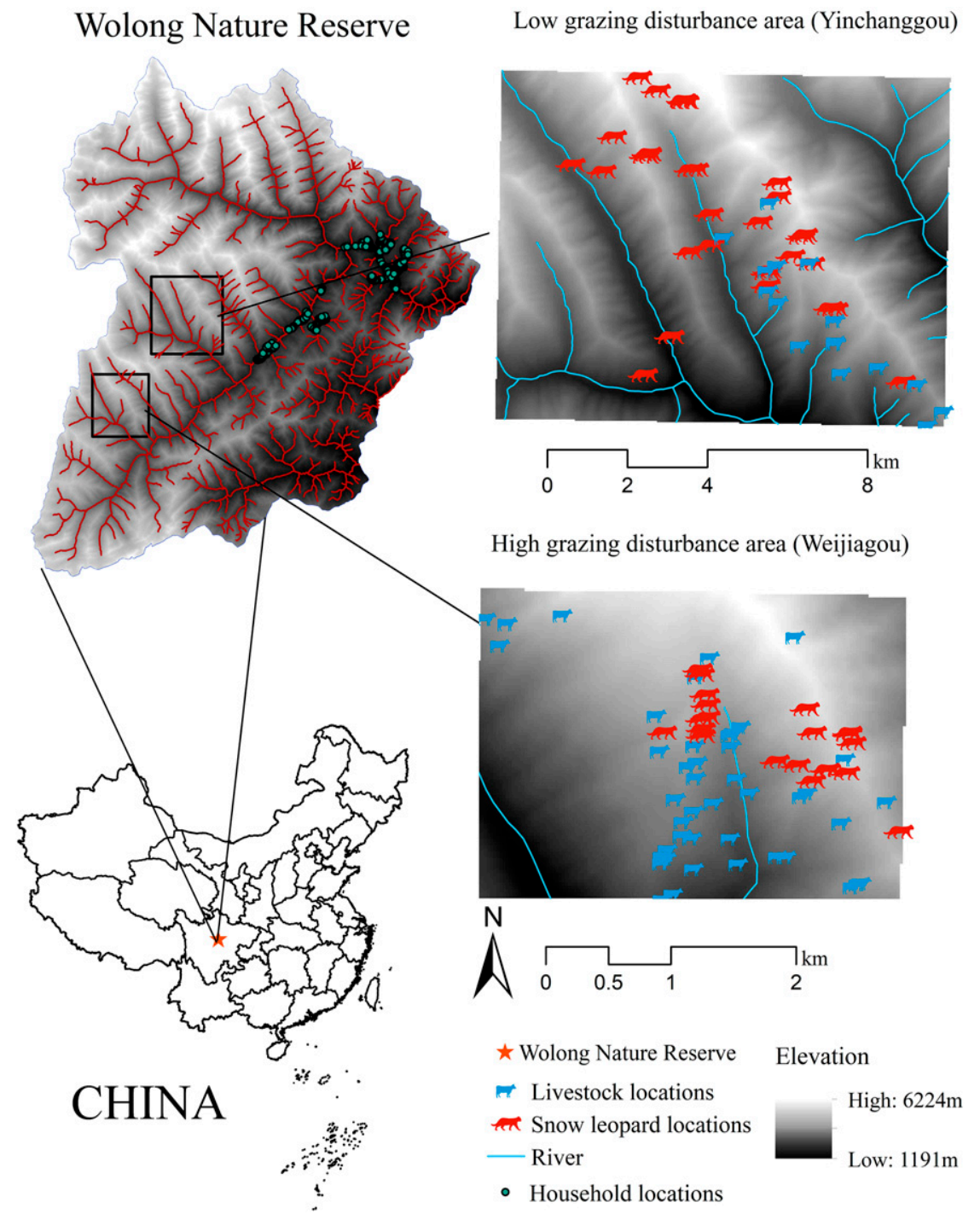

FIG. 1. Map and location of Wolong Nature Reserve in Sichuan. Livestock and snow leopard locations in LGDAs and HGDAs are also depicted.

In the process of walking line transects, we slightly adjusted their direction to avoid dangerous conditions. All members of the investigation team were trained to identify the signs of snow leopards before the survey began. If snow leopard sign was found along the line transect, we then set up a sample plot $(20 \mathrm{~m} \times 20 \mathrm{~m})$ around it. Each potential sign was photographed and associated samples (e.g., feces and hair) were brought back to China West Normal University. The "Snow Leopard Survey Technology Manual" jointly formulated by Peking University and Shanshui Nature Conservation Center and "A Guide to the Mammals of China" and the corresponding references (Anwar et al. 2011; Ma et al. 2005; Xu et al. 2005; Liu et al. 2003) were then used to confirm the collected signs as those of snow leopards. Invited mammalogy experts from China West Normal University and the Key Laboratory of Southwest China Wildlife Resources Conservation (China West Normal University) confirmed whether the photographs and samples were signs of snow leopards. In addition to the snow leopard plots, we set up control plots in areas where there were no signs of snow leopards. We established these plots in a random manner so that the minimum distance between two plots was $500 \mathrm{~m}$, and the maximum distance was not more than $600 \mathrm{~m}$. We employed this spacing interval to reduce the influence of excessive changes in environmental conditions between two plots on the analysis.

We established a total of 142 sample plots in the summer 2019 and 2020, with 92 plots (44 observation plots and 48 control sample plots) in LGDAs and 50 plots (18 observation plots and 32 control sample plots) in HGDAs. Each $20 \mathrm{~m} \times$ $20 \mathrm{~m}$ plot contained 4 shrub subplots $(10 \mathrm{~m} \times 10 \mathrm{~m})$ and 3 herb subplots $(1 \mathrm{~m} \times 1 \mathrm{~m})$ (Fig. 2$)$. We measured several microhabitat factors in each main plot, including the elevation, slope $\left(0^{\circ}-5^{\circ}, 6^{\circ}-15^{\circ}, 16^{\circ}-30^{\circ}, 31^{\circ}-45^{\circ}, 46^{\circ}-60^{\circ}\right.$, and $\left.\geq 61^{\circ}\right)$, slope aspect (north, northeast, east, southeast, south, southwest, west, and northwest), slope location (ridge, the bottom of the slope, the 

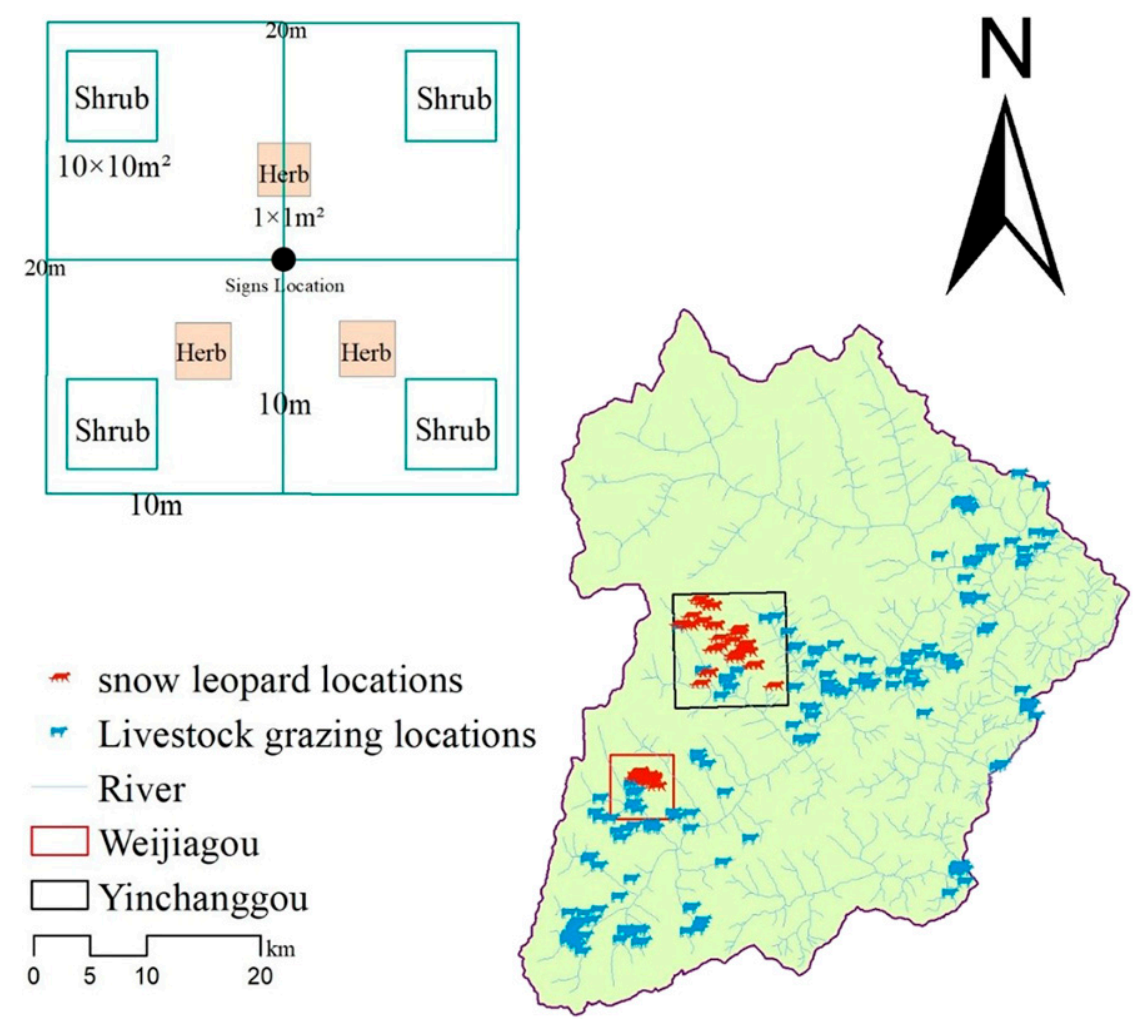

FIG. 2. Livestock grazing and snow leopard locations in Wolong Nature Reserve. The inset is the study design that we used for individual plots.

middle of the slope, and the top of the slope), terrain ruggedness (flat, rugged, lightly steep, middling steep, and highly steep), and vegetation type (mixed broadleaf, shrub forest, meadow, and alpine screes). We counted the number of shrub species and the basal diameter, height, number, and coverage of shrubs in the shrub subplots and the number of herb species and the height and coverage of herbs in the herb subplots.

\section{b. Data analysis}

We used the nearest point index $R$ and geographical concentration index $G$ (Yang et al. 2019) to understand the distribution and degree of concentration of snow leopards in LGDAs and HGDAs. The nearest point index formula is as follows:

$$
R=\frac{\bar{d}_{\min }}{E\left(d_{\min }\right)}
$$

where $\bar{d}_{\min }$ is the average of the observed nearest point distance among snow leopard sites and $E\left(d_{\min }\right)$ is the average of the theoretical nearest point distance between a random distribution of sites. An $R$ value equal to 0 indicates a uniform distribution of points, $0<R<1$ indicates an aggregated distribution, and an $R$ value equal to 1 indicates a random distribution.

To calculate $E\left(d_{\min }\right)$, we used the following formula:

$$
E\left(d_{\min }\right)=\frac{1}{2 \sqrt{n / A}},
$$

where $n$ is the number of subjects in the corresponding plot and $A$ is the acreage of the plot. We used ArcGIS10.2 software to get the nearest point distances and calculated the averages in Microsoft Excel 2010. We also used the geographical concentration index:

$$
G=100 \times \sqrt{\sum_{i=1}^{n}\left(X_{i} / T\right)^{2}},
$$

where $X_{i}$ is the number of subjects in plot $i, T$ is the total number of subjects, and $n$ is the number of plots. The possible range of $G$ values is $0-100$, with larger values indicating greater degrees of concentration.

Kolmogorov-Smirnov (K-S) tests showed that our continuous variables were normally distributed, and we thus conducted independent-samples $t$ tests to test for significant differences in elevation and vegetation factors between LGDAs and HGDAs. Vegetation factors included the number of shrub species, basal diameter, height, number, and coverage of shrubs and the number of herb species, height, and coverage of herbs. We conducted nonparametric (MannWhitney) tests to examine the differences in slope, slope aspect, slope location, terrain ruggedness, and vegetation type between LGDAs and HGDAs. We conducted a chi-square test to examine whether snow leopards were selective for certain values of five categorical variables (slope, slope aspect, slope location, terrain ruggedness, and vegetation type) in LGDAs and HGDAs, respectively. 
Resource selection functions (RSF) are commonly used to study the relationship between wildlife and their habitat (Gillies et al. 2010). We built RSFs using generalized linear models in LGDAs and HGDAs separately according to the following formula:

$$
g\left(\mu_{i}\right)=\beta_{0}+\beta_{1} x_{1}+\beta_{1} x_{2}+\cdots+\beta_{i} x_{i}+\cdots+\beta_{n} x_{n},
$$

where $g\left(\mu_{i}\right)$ is the relative probability of selection and $\beta_{n}$ is the coefficient for the $n$th predictive environmental variable $x_{n}$ (Bai et al. 2020). Before building the model, all continuous variables were standardized according to zero-mean normalization. We also excluded variables so that no pair of variables had a Pearson correlation coefficient greater than 0.6. The variables shrub height and number of shrub species were omitted in the RSF of LGDAs, and the shrub height, shrub cover, and number of shrub species were deleted in the RSF of HGDAs. We modeled the relative probability of selection as a binomial distribution with a logistic link function. The response variable in our RSF was coded as snow leopard detection locations (1) and snow leopard nondetection locations (0). We present results from the most-supported model. The $t$ tests and Mann-Whitney tests were conducted with SPSS 16.0 software, and building the resource selection models was conducted with $\mathrm{R}$ 4.0.2 software. For all statistical analyses, we set the significance level to 0.05 .

\section{Results}

\section{a. Spatial utilization in LGDAs and HGDAs}

The sign density of snow leopards and blue sheep in HGDAs was larger than that in LGDAs. Specifically, these species had sign densities of 0.45 and $1.39 \mathrm{~km}^{2}$ in LGDAs and sign densities of 2.50 and $7.75 \mathrm{~km}^{2}$ in HGDAs, respectively. Higher sign densities do not necessarily indicate a larger number of individuals - it may just be a reflection of the activity of the populations being more concentrated. Snow leopards, livestock, and blue sheep all featured aggregated distributions in both LGDAs and HGDAs (Table 1), but the distribution of snow leopards in HGDAs $(G=61.68)$ was more concentrated than that in LGDAs $(G=80.13)$.

\section{b. Microhabitat-use differences between LGDAs and HGDAs}

We analyzed a total of 14 habitat characteristics, and 6 were significantly different between LGDAs and HGDAs $(p<$ $0.05)$. We found greater height and coverage of herbs but lower herb species diversity in LGDAs. The slope, slope aspect and vegetation type were also significantly different between LGDAs and HGDAs (Table 2). Relative to the LGDAs, there was an additional slope category with snow leopard sign in HGDAs: greater than or equal to $61^{\circ}\left(\geq 61^{\circ}\right)$. Relative to the HGDAs, there were additional slope aspects containing snow leopard sign (east and northwest) and additional vegetation types (mixed broadleaf-conifer forest and shrub forest) in LGDAs.

According to the chi-square test, we also found that the selection of snow leopard in response to five categorical
TABLE 1 . The nearest point index $R$ result and distribution type of species in LGDAs and HGDAs.

\begin{tabular}{|c|c|c|c|c|}
\hline Species & $\bar{d}_{\min }$ & $E\left(d_{\min }\right)$ & $R$ & Distribution type \\
\hline \multicolumn{5}{|l|}{ LGDAs } \\
\hline Snow leopard & 0.0019 & 0.5959 & 0.0032 & Aggregated \\
\hline Livestock & 0.0055 & 0.6472 & 0.0081 & Aggregated \\
\hline Blue sheep & 0.0012 & 0.3223 & 0.0037 & Aggregated \\
\hline \multicolumn{5}{|l|}{ HGDAs } \\
\hline Snow leopard & 0.0012 & 0.1704 & 0.007 & Aggregated \\
\hline Livestock & 0.0013 & 0.1913 & 0.0067 & Aggregated \\
\hline Blue sheep & 0.0012 & 0.1788 & 0.0067 & Aggregated \\
\hline
\end{tabular}

variables was significant in LGDAs but not in HGDAs (Table 3). In other words, there was more selectivity in the slope, slope aspect, slope location, terrain ruggedness, and vegetation type variables by snow leopards in LGDAs.

\section{c. Microhabitat selection in LGDAs and HGDAs}

In LGDAs, the final model predicting microhabitat selection of snow leopards was significantly affected by elevation, shrub basal diameter, and shrub height $(p<0.05)$. Snow leopards preferentially selected higher elevations, areas with shrubs that had smaller basal diameter, and areas where herb height was lower (Table 4; Fig. 3). In HGDAs, the final model included no significant variables predicted to affect the microhabitat selection of snow leopards (Table 4).

\section{Discussion}

The rapid development of livestock rearing is profoundly affecting natural ecosystems (e.g., freshwater systems, forests, grasslands) around the world (Liu et al. 2015). With increases in the population and activity range of livestock, there has been corresponding loss and fragmentation of many rare wildlife's habitat. This is the case even for species that live at high elevation areas, such as snow leopard and blue sheep (Shi et al. 2019; Khan et al. 2016). Some studies have found that the distribution of snow leopards is dependent on that of blue sheep, their main prey species (J. Li et al. 2020; Alexander et al. 2016b). With livestock occupying the space and food resources of blue sheep, their viability in the ecosystem decreases (Karimov et al. 2018). Although some studies in recent years have shown that livestock make up an increasing proportion of the snow leopard's diet, the snow leopard's main food source is still blue sheep (Oli et al. 1993; Bagchi and Mishra 2006; Lu et al. 2019; Shehzad et al. 2012). The availability of this prey is an important factor affecting the habitat selection of snow leopard (J. Li et al. 2020; Alexander et al. 2016b; Mosheh and Som 2009). As space utilization of blue sheep is reduced under the impact of livestock, the space available for snow leopards is in turn smaller. Because there are more human activities in HGDAs such as feeding livestock with salt, medicine, and shearing, snow leopards may also avoid specific areas with increased human activity there (Wolf and Ale 2009). We posit that snow leopard sign was more concentrated in HGDAs for this reason. 
TABLE 2. Difference tests ( $t$ test and Mann-Whitney test) comparing habitat characteristics between LGDAs and HGDAs; SD is standard deviation. An asterisk indicates significant differences $(p<0.05)$.

\begin{tabular}{|c|c|c|c|c|}
\hline Habitat characteristics & Mean \pm SD LGDAs & Mean \pm SD HGDAs & $t$ & $p$ \\
\hline \multicolumn{5}{|c|}{$t$ test } \\
\hline Elev (m) & $4131.98 \pm 43.59$ & $4058.61 \pm 26.83$ & 1.433 & 0.157 \\
\hline No. of shrub species & $2.00 \pm 0.37$ & $1.36 \pm 0.15$ & 1.568 & 0.133 \\
\hline Basal diam of shrubs $(\mathrm{cm})$ & $2.85 \pm 0.77$ & $1.95 \pm 0.71$ & 0.790 & 0.437 \\
\hline Height of shrubs $(\mathrm{cm})$ & $33.64 \pm 9.07$ & $20.14 \pm 2.09$ & 1.450 & 0.165 \\
\hline No. of shrubs & $8.80 \pm 3.47$ & $9.68 \pm 2.25$ & -0.085 & 0.933 \\
\hline Coverage of shrubs (\%) & $31.35 \pm 6.89$ & $21.00 \pm 3.82$ & 1.315 & 0.201 \\
\hline No. of herb species & $5.13 \pm 0.40$ & $6.22 \pm 0.34$ & -2.074 & $0.043^{*}$ \\
\hline Coverage of herbs (\%) & $55.74 \pm 4.89$ & $42.28 \pm 4.50$ & 2.027 & $0.048^{*}$ \\
\hline Height of herbs & $12.55 \pm 1.12$ & $4.13 \pm 0.48$ & 6.870 & $0.000^{*}$ \\
\hline \multicolumn{5}{|c|}{ Mann-Whitney test } \\
\hline Slope & - & - & - & $0.024^{*}$ \\
\hline Slope aspect & - & - & - & $0.027^{*}$ \\
\hline Slope location & - & - & - & 0.322 \\
\hline Terrain ruggedness & - & - & - & 0.150 \\
\hline Vegetation type & - & - & - & $0.013^{*}$ \\
\hline
\end{tabular}

In addition to the availability of prey and human disturbance (Sharma et al. 2015), there are many factors affecting the habitat selection of snow leopards (J. Li et al. 2020), including elevation (Alexander et al. 2016b), terrain (Sharma et al. 2015), and vegetation type (Tang et al. 2017). We used RSFs to investigate the effects of multiple variables on the habitat selection of snow leopards. Elevation has always been an important environmental variable in the study of snow leopard habitat selection. Numerous studies have shown that snow leopards tend to choose higher elevations (Tang et al. 2017; Qiao et al. 2017; Xu et al. 2006), and we also obtained this result in our RSF. As elevation increases, vegetation cover gradually decreases and is replaced by alpine screes. Snow leopards are opportunistic predators (Maheshwari and Sathyakumar 2020; J. Li et al. 2020) that camouflage themselves by using environmental factors to improve the success rate of predation. The unique body color and pattern of snow leopards allow snow leopards to better hide in the alpine scree environment of high elevations. In addition to this, high elevations are often accompanied by steep and rugged terrain that the unique body structure of snow leopards is well suited for in hunting prey species. More important, there are relatively few large carnivore species distributed in high elevation areas, which reduces competition for resources (Hong et al. 2020).
At present, there are few studies on the selection of vegetation type and structure by snow leopards. Potential reasons for this include the following: 1) Snow leopards are carnivores and do not directly consume plant material-therefore, the influence of vegetation on snow leopards has been ignored. 2) Plant communities and vegetation structure in the high elevation ecosystem are relatively simple. There is little change in vegetation between different areas, likely resulting in a lack of interest in vegetation research by snow leopard researchers. However, with the continuous expansion of livestock grazing in recent years (Wang et al. 2019), its impact on the vegetation of high-elevation ecosystems has become increasingly significant. Different grazing intensities have different effects on vegetation in different regions. Therefore, our study fills an important knowledge gap concerning the vegetation selection of snow leopards in areas of different grazing intensities.

According to our RSF, we found that snow leopards tended to choose areas in which shrubs had smaller basal diameters and herbs had lower heights. Snow leopards not only use alpine screes habitats, but they also use alpine meadow habitats (Hong et al. 2020; J. Li et al. 2020). Snow leopards living in alpine meadows still need to hide when hunting prey, and shrubs with smaller basal diameter can provide good hiding

TABLE 3. The chi-square test of five categorical variables in LGDAs and HGDAs, separately; df indicates degrees of freedom. An asterisk denotes significant differences $(p<0.05)$.

\begin{tabular}{lcccccc}
\hline \hline & \multicolumn{3}{c}{ LGDAs } & & \multicolumn{2}{c}{ HGDAs } \\
\cline { 2 - 5 } Categorical variables & Chi-square & df & $p$ & & Chi-square & df \\
\hline Slope & 13.273 & 3 & $0.004^{*}$ & 3.667 & 4 & 0.453 \\
Slope aspect & 12.182 & 5 & $0.032^{*}$ & 6.444 & 3 & 0.092 \\
Slope location & 45.773 & 4 & $0.000^{*}$ & 2.333 & 2 & 0.311 \\
Terrain ruggedness & 28.909 & 3 & $0.000^{*}$ & 1.000 & 2 & 0.607 \\
Vegetation type & 22.591 & 4 & $0.000^{*}$ & 2.130 & 0.144 \\
\hline
\end{tabular}


TABLE 4. The factors influencing the microhabitat selection of snow leopard through binary logistic regression (variables in the equation) in LGDAs and HGDAs. One and two asterisks denote contributions to the model that are significant at $p<0.05$ and $p<0.01$, respectively.

\begin{tabular}{|c|c|c|c|c|}
\hline Factors & Estimate & Std error & $Z$ value & $\operatorname{Pr}(>|z|)$ \\
\hline \multicolumn{5}{|l|}{ LGDAs } \\
\hline Intercept & -12.5294 & 5.3363 & -2.348 & $0.0189^{*}$ \\
\hline Elev & 0.0038 & 0.0013 & 2.921 & $0.0035^{* *}$ \\
\hline Basal diam of shrubs & -0.3979 & 0.1211 & -3.286 & $0.0010^{* *}$ \\
\hline Height of herbs & -0.0856 & 0.0397 & -2.155 & $0.0312^{*}$ \\
\hline \multicolumn{5}{|l|}{ HGDAs } \\
\hline Intercept & -1.4386 & 561.7403 & -0.003 & 0.9979 \\
\hline Height of herbs & -0.3253 & 0.1911 & -1.702 & 0.0887 \\
\hline No. of shrubs & -1.978 & 0.1109 & -1.783 & 0.0746 \\
\hline Slope location (the bottom of slope) & -16.5158 & 1507.3048 & -0.011 & 0.9913 \\
\hline Slope location (the middle of slope) & -6.0120 & 1123.4784 & -0.005 & 0.9957 \\
\hline Slope location (the top of slope) & -3.5750 & 502.4352 & -0.007 & 0.9943 \\
\hline
\end{tabular}

conditions and will not overly hinder snow leopard movement when chasing prey (Hong and Zhang 2021). The selection of areas with lower herbs may be related to the fact that snow leopards are top predators in the high elevation ecosystem and thus do not need to avoid predators themselves. When snow leopards are not hunting, they might then choose areas with lower herb heights as it costs less energy to move around as compared with areas with higher herb heights. Lower herbs might also enhance the field of vision for snow leopards surveying for prey.

Our results showed that elevation, basal diameter of shrubs and herb height influenced the microhabitat selection of snow leopard in LGDAs and that no factors significantly affected the microhabitat selection of snow leopard in HGDAs. Together with the results of our chi-square tests of categorical habitat factors and snow leopard presence, this indicates that snow leopards may be more selective of environmental factors under low disturbance conditions, while high human disturbance might limit their potential to select for preferred habitat. We also found that the height and coverage of herbs, and thus overall herb biomass, decreased with high livestock sign density $\left(5.84 \mathrm{~km}^{2}\right)$ (Table 2$)$. This reduces the forage available to wild ungulates like blue sheep, likely leading to smaller populations there and in turn less prey availability for snow leopards.

\section{Conclusions}

Our study confirmed that livestock grazing has significant impacts on the distribution and microhabitat selection of snow leopards and explained the possible reasons for these effects. We recommend that grazing intensity be controlled with the aim of restoring the habitat complexity and diversity of the alpine environments frequented by snow leopards. This will allow the distribution of the snow leopards to remain more dispersed and allow for more selectivity of habitat factors. By controlling the area and intensity of livestock grazing, direct human-snow leopard conflict can also be minimized. We believe this study is valuable for improving the management of livestock and wildlife and that similar research should be considered in other reserves.

Acknowledgments. The authors thank H. K. Yin, J. Hou, T. G. Cai, and Z. E. Mao from China West Normal university for assistance with data collection. We thank Dr. W. K. Bai for his guidance on the editing of the paper. This study was financially supported by the following sources of funding: the Second Tibetan Plateau Scientific Expedition and Research Program (STEP) (Grant 2019QZKK0402), the National Natural Science Foundation of China (42071279, 31801991, 41571517, 31572293), the Key Laboratory of Southwest China Wildlife Resources Conservation (China West Normal University), Ministry of Education of China (XNYB17-2, XNYB19-01), Research innovation team funding project of China West Normal University (CXTD2018-9), 2020 College Students' Innovative Entrepreneurial Training Plan program of China West Normal University (S202010638084X, S202010638087), and the fund of China West Normal University (YC358, 17E073, 17E074).
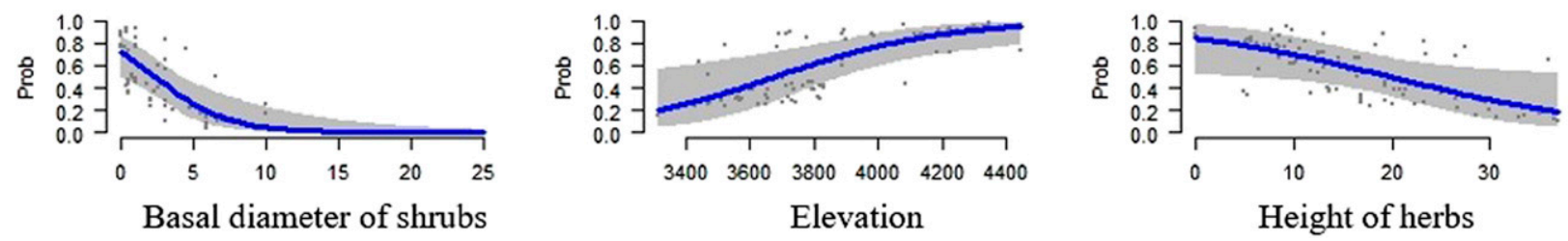

FIG. 3. Relative probability of selection for the factors in the optimal resource selection function in LGDAs. 


\section{REFERENCES}

Alexander, J. S., A. M. Gopalaswamy, K. Shi, J. Hughes, P. Riordan, and E. Z. Cameron, 2016a: Patterns of snow leopard site use in an increasingly human-dominated landscape. PLOS ONE, 11, e0155309, https://doi.org/10.1371/ journal.pone.0155309.

— K. Khi, L. A. Tallents, and P. Riordan, 2016b: On the high trail: Examining determinants of site use by the endangered snow leopard Panthera uncia in Qilianshan, China. Oryx, 50, 231-238, https://doi.org/10.1017/S0030605315001027.

Anwar, M. B., R. Jackson, M. S. Nadeem, J. E. Janeka, S. Hussain, M. A. Beg, G. Muhammad, and M. Qayyum, 2011: Food habits of the snow leopard Panthera uncia (Schreber, 1775) in Baltistan, northern Pakistan. Eur. J. Wildl. Res., 57, 1077 1083, https://doi.org/10.1007/s10344-011-0521-2.

Bagchi, S., and C. Mishra, 2006: Living with large carnivores: Predation on livestock by the snow leopard (Uncia uncia).J. Zool., 268, 217224, https://doi.org/10.1111/j.1469-7998.2005.00030.x.

Bai, W. K., 2017: The dynamics of space utilization and habitat selection of giant pandas (Ailuropoda melanoleuca) in Wolong Nature Reserve. Ph.D. thesis, Inner Mongolia Agricultural University, Q958.1, 94 pp., https:/wap.cnki.net/touch/web/ Dissertation/Article/10129-1017212351.nh.html.

— , and Coauthors, 2020: Microhabitat selection by giant pandas. Biol. Conserv., 247, 108615, https://doi.org/10.1016/j.biocon.2020.108615.

Bargali, H. S., T. Ahmed, and G. Umapathy, 2018: Patterns of livestock depredation by tiger (Panthera tigris) and leopard (Panthera pardus) in and around Corbett Tiger Reserve, Uttarakhand, India. PLOS ONE, 13, e0195612, https:// doi.org/10.1371/journal.pone.0195612.

Berger, J., B. Buuveibaatar, and C. Mishra, 2013: Globalization of the cashmere market and the decline of large mammals in central Asia. Conserv. Biol., 27, 679-689, https://doi.org/ 10.1111/cobi.12100.

Cheng, Y. H., and Coauthors, 2015: Investigation of exotic plants in Wolong National Nature Reserve. J. Sichuan For. Sci. Technol., 36, 125-132, https://doi.org/10.16779/j.cnki.1003-5508.2015.03.028.

Dickman, A. J., 2009: Key determinants of conflict between people and wildlife, particularly large carnivores, around Ruaha National Park, Tanzania. Ph.D. thesis, University College London, $373 \mathrm{pp}$.

Farrington, J. D., and J. Li, 2016: Climate change impacts on snow leopard range. Snow Leopards, T. McCarthy and D. Mallon, Eds., Academic Press, 85-95.

Forrest, J. L., and Coauthors, 2012: Conservation and climate change: Assessing the vulnerability of snow leopard habitat to treeline shift in the Himalaya. Biol. Conserv., 150, 129-135, https://doi.org/10.1016/j.biocon.2012.03.001.

Ghoshal, A., Y. Bhatnagar, B. Pandav, K. Sharma, C. Mishra, R. Raghunath, and K. Suryawanshi, 2019: Assessing changes in distribution of the endangered snow leopard Panthera uncia and its wild prey over 2 decades in the Indian Himalaya through interview-based occupancy surveys. Oryx, 53, 620 632, https://doi.org/10.1017/S0030605317001107.

Gillies, C. S., and Coauthors, 2010: Application of random effects to the study of resource selection by animals. J. Anim. Ecol., 75, 887-898, https://doi.org/10.1111/j.1365-2656.2006.01106.x.

Hong, Y., and J. D. Zhang, 2021: Habitat selection and food source of snow leopard (Panthera uncia) in Wolong National Nature Reserve, Sichuan Province, China. Yesheng Dongwu, 42, 295-305.

- - - and Y. J. Wang, 2020: Progress in the ecology and conservation research of Panthera unica. Sichuan J. Zool., 39, 711-720, https://doi.org/10.11984/j.issn.1000-7083.20190438.
Hou, J., J. Yang, Y. J. Li, J. Y. Huang, D. Liu, and J. D. Zhang, 2018: Spatio-temporal distribution characteristics of mammals investigated using infrared camera traps in Wolong Nature Reserve. J. Nanjing Forest. Univ., 42, 187-192.

Hull, V., and Coauthors, 2014: Impact of livestock on giant pandas and their habitat. J. Nat. Conserv., 22, 256-264, https://doi.org/ 10.1016/j.jnc.2014.02.003.

Jackson, R., 2002: Snow leopard status, distribution and protected areas coverage: A report. Snow Leopard Survival Strategy Rep., 24 pp., https://www.snowleopardnetwork.org/bibliography/ Jackson_2002.pdf.

Johansson, Ö., T. McCarthy, G. Samelius, H. Andrén, L. Tumursukh, and C. Mishra, 2015: Snow leopard predation in a livestock dominated landscape in Mongolia. Biol. Conserv., 184, 251-258, https://doi.org/10.1016/j.biocon.2015.02.003.

Karimov, K., S. M. Kachel, K. Hacklnder, and F. B. Marco, 2018: Responses of snow leopards, wolves and wild ungulates to livestock grazing in the Zorkul Strictly Protected Area, Tajikistan. PLOS ONE, 13, e0208329, https://doi.org/10.1371/ journal.pone.0208329.

Khan, B., A. Ablimit, G. Khan, A. W. Jasra, H. Ali, R. Ali, E. Ahmad, and M. Ismail, 2016: Abundance, distribution and conservation status of Siberian ibex, Marco Polo and Blue sheep in Karakoram-Pamir mountain area. J. King Saud Univ. Sci., 28, 216-225, https://doi.org/10.1016/ j.jksus.2015.02.007.

Koziarski, A., B. Kissui, and C. Kiffner, 2016: Patterns and correlates of perceived conflict between humans and large carnivores in northern Tanzania. Biol. Conserv., 199, 41-50, https:// doi.org/10.1016/j.biocon.2016.04.029.

Landa, A., K. Gudvangen, J. E. Swenson, and E. Røskaft, 1999: Factors associated with wolverine Gulo gulo predation on domestic sheep. J. Appl. Ecol., 36, 963-973, https://doi.org/ 10.1046/j.1365-2664.1999.00451.x.

Li, B. B., R. Kelley, S. Nitya, C. Liu, and K. K. Krithi, 2020: Effects of livestock loss and emerging livestock types on livelihood decisions around protected areas: Case studies from China and India. Biol. Conserv., 248, 108645, https://doi.org/10.1016/ j.biocon.2020.108645.

Li, J., Y. W. Ma, N. Jiang, H. Yang, H. X. Zhou, Y. R. Wu, and Y. H. Wu, 2020: Research progress in conservation biology of snow leopard (Panthera uncia). Yesheng Dongwu, 41, 796805 .

Li, S., J. M. William, D. J. Wang, X. D. Gu, and X. L. Shen, 2020: Retreat of large carnivores across the giant panda distribution range. Nat. Ecol. Evol., 4, 1327-1331, https://doi.org/10.1038/ s41559-020-1260-0.

Liu, C. G., S. W. Zheng, and J. R. Ren, 2003: Research foods and foods source about snow leopard (Panthera uncia). J. Shaanxi Norm. Univ., S2, 154-159.

Liu, J., H. Miao, H. Zheng, Z. Y. Ouyang, X. K. Wang, X. G. Li, and B. Jiang, 2009: Discussion about the relationship pattern between Wolong Nature Reserve and local community. Acta Ecol. Sin., 29, 259-271.

— , and Coauthors, 2015: Systems integration for global sustainability. Science, 347, 1258832, https://doi.org/10.1126/science.1258832.

Liu, P. J., and H. D. Han, 2015: The status quo, influence and countermeasures of snow leopard population in Tomuer Peak Nature Reserve. Anhui Nongye Kexue, 5, 103-104.

Lu, Q., Q. Hu, X. G. Shi, S. L. Jin, S. Li, and M. Yao, 2019: Metabarcoding diet analysis of snow leopard (Panthera uncia) in Wolong National Nature Reserve, Sichuan Province. Biol. Diversity, 27, 960-969, https://doi.org/10.17520/biods.2019101. 
Lukarevskiy, V. S., M. Dalannast, S. Lukarevskiy, and E. Damdin, 2019: Factors determining the distribution and status of the snow leopard population (Panthera uncia) in Western Mongolia. Anim. Vet. Sci., 7, 127-132, https://doi.org/10.11648/ j.avs.20190706.12.

Ma, M., B. Munkhtsog, F. Xu, M. Turghan, S. J. Yin, and S. D. Wei, 2005: Markings as indicator of snow leopard in field survey, in Xinjiang. J. Zool., 40, 34-39.

Maheshwari, A., and S. Sathyakumar, 2020: Patterns of livestock depredation and large carnivore conservation implications in the Indian Trans-Himalaya. J. Arid Environ., 182, 104241, https://doi.org/10.1016/j.jaridenv.2020.104241.

McCarthy, T., D. Mallon, R. Jackson, P. Zahler, and K. McCarthy, 2017: Panthera uncia. IUCN Red List of Threatened Species, e.T22732A50664030, https://doi.org/10.2305/IUCN.UK.20172.RLTS.T22732A50664030.en.

Morell, V., 2017: Rare snow leopard's diet is one-quarter livestock. Science, https://doi.org/10.1126/science.aal0723.

Oli, M. K., I. R. Taylor, and D. M. E. Rogers, 1993: Diet of the snow leopard (Panthera uncia) in the Annapurna Conservation Area, Nepal. J. Zool., 231, 365-370, https://doi.org/10.1111/ j.1469-7998.1993.tb01924.x.

Qiao, M. J., Z. Tang, X. G. Shi, Y. H. Cheng, Q. Hu, W. J. Li, and H. M. Zhang, 2017: Habitat suitability assessment of snow leopard in Wolong National Nature Reserve based on MaxEnt modeling. J. Sichuan For. Sci. Technol., 38, 1-4, 16, https:// doi.org/10.16779/j.cnki.1003-5508.2017.06.001.

Rovero, F., C. Augugliaro, R. S. Havmoller, C. Groff, F. Zimmermann, V. Oberosler, and S. Tenan, 2020: Co-occurrence of snow leopard panthera uncia, Siberian ibex capra sibirica and livestock: Potential relationships and effects. Oryx, 54, 118124, https://doi.org/10.1017/S0030605317001685.

Sandeep, S., T. Kamal, K. C. Mukesh, and D. Trishna, B. YashVeer, and M. M. Thomas, 2006: The snow leopard in Himalaya: A step towards conservation by studying their distribution, marking, habitat selection, coexistence with other predators, and wild prey-livestock-predator interaction. Conservation Biology in Asia, J. A. McNeely, et al., Eds., Society of Conservation Biology Asia Section and Resources Himalaya Foundation, 184-196.

Sharma, R. K., Y. V. Bhatnagar, and C. Mishra, 2015: Does livestock benefit or harm snow leopard? Biol. Conserv., 190, 8-13, https://doi.org/10.1016/j.biocon.2015.04.026.

Shehzad, W., T. M. McCarthy, F. Pompanon, L. Purevjav, E. Coissac, T. Riaz, P. Taberlet, and R. DeSalle, 2012: Prey preference of snow leopard (Panthera uncia) in South Gobi, Mongolia. PLOS ONE, 7, e32104, https://doi.org/10.1371/ journal.pone.0032104.

Shi, X. G., Q. Hu, J. Q. Li, Z. Tang, J. Yang, W. J. Li, X. L. Shen, and S. Li, 2017: Camera-trapping surveys of the mammal and bird diversity in Wolong National Nature Reserve, Sichuan Province. Biol. Diversity, 25, 1131-1136, https://doi.org/ 10.17520/biods.2017193.

Shi, X. Y., X. G. Shi, Q. Hu, T. P. Guan, Q. Fu, J. Zhang, M. Yao, and S. Li, 2019: Evaluating the potential habitat overlap and predation risk between snow leopard and free-range yaks in the Qionglai Mountains, Sichuan. Biol. Diversity, 27, 951-959, https://doi.org/10.17520/biods.2019062.

Suryawanshi, K. R., Y. V. Bhatnagar, S. Redpath, C. Mishra, and N. Pettorelli, 2013: People, predators and perceptions: Patterns of livestock depredation by snow leopard and wolves. J. Appl. Ecol., 50, 550-560, https://doi.org/10.1111/ 1365-2664.12061.

—, S. M. Redpath, Y. V. Bhatnagar, U. Ramakrishnan, V. Chaturvedi, S. C. Smout, and C. Mishra, 2017: Impact of wild prey availability on livestock predation by snow leopard. Roy. Soc. Open Sci., 4, 170026, https://doi.org/10.1098/ rsos.170026.

Tang, Z., J. Yang, X. H. Liu, P. Y. Wang, and Z. Y. Li, 2017: Research on snow leopard (Panthera uncia) using cameratrapping in Wolong National Nature Reserve, China. Biol. Diversity, 25, 62-70, https://doi.org/10.17520/biods.2016313.

Thinley, P., Dorji, C. Namgyel, S. Yoenten, Dorji, 2014: Estimating snow leopard (Panthera uncia) abundance and distribution in Jigme Dorji National Park using camera traps: A technical report. KUENSEL Corporation Ltd. Tech. Rep., 45 pp.

Wang, F., and Coauthors, 2021: The hidden risk of using umbrella species as conservation surrogates: A spatio-temporal approach. Biol. Conserv., 253, 108913, https://doi.org/10.1016/ j.biocon.2020.108913.

Wang, X., J. Hou, J. D. Zhang, W. K. Bai, J. Y. Huang, S. Q. Zhou, and Z. Y. Ouyang, 2018a: Divergent behavioral responses of sympatric species to grazing disturbance. Acta Ecol. Sin., 38, 6484-6492.

_, Y. J. Li, C. Li, and J. D. Zhang, 2018b: The impact of livestock on giant pandas in Wolong Nature Reserve. J. China West Norm. Univ., 39, 11-15.

— versity and giant panda habitat. J. Wildl. Manage., 83, 15921597, https://doi.org/10.1002/jwmg. 21743.

Wei, W., and Coauthors, 2018: Giant panda distributional and habitat-use shifts in a changing landscape. Conserv. Lett., 11, e12575, https://doi.org/10.1111/conl.12575.

Wolf, M., and S. Ale, 2009: Signs at the top: Habitat features influencing snow leopard Uncia uncia activity in Sagarmatha National Park, Nepal. J. Mammal., 90, 604-611, https:// doi.org/10.1644/08-MAMM-A-002R1.1.

Xu, F., M. Ma, S. J. Yin, and M. Turghan, 2005: Snow leopard survey in tumor nature reserve, Xinjiang. Sichuan Dong $W u$, 24, 608-610.

,,--- , and M. Bariushaa, 2006: Autumn habitat selection by snow leopard (Uncia uncia) in Beita Mountain, Xinjiang, China. Zool. Res., 27, 221-224.

Yang, N., Y. Li, and Y. L. Huang, 2019: Spatial distribution characteristics analysis of GIS-related academic research institutes in the USA. Sci. Surv. Mapp., 4, 188-195.

Zhang, J. D., and Coauthors, 2017: Divergent responses of sympatric species to livestock encroachment at fine spatiotemporal scales. Biol. Conserv., 209, 119-129, https://doi.org/10.1016/ j.biocon.2017.02.014.

—, T. Connor, H. B. Yang, Z. Y. Ouyang, S. Li, and J. G. Liu, 2018: Complex effects of natural disasters on protected areas through altering telecouplings. Ecol. Soc., 23, 17, https:// doi.org/10.5751/ES-10238-230317.

, J. Y. Huang, S. Q. Zhou, V. Hull, H. M. Zhang, and Z. Y. Ouyang, 2019: Spatial utilization and activity pattern characteristics of a giant panda during the reproduction-rearing period. Shoulei Xuebao, 4, 421-430.

Zhang, Y. S., Z. X. Su, and J. Y. Hu, 2005: Spatial distribution pattern of Davidia involucrata population in Wolong of Sichuan Province. Yunnan Zhiwu Yanjiu, 27, 395-402. 